
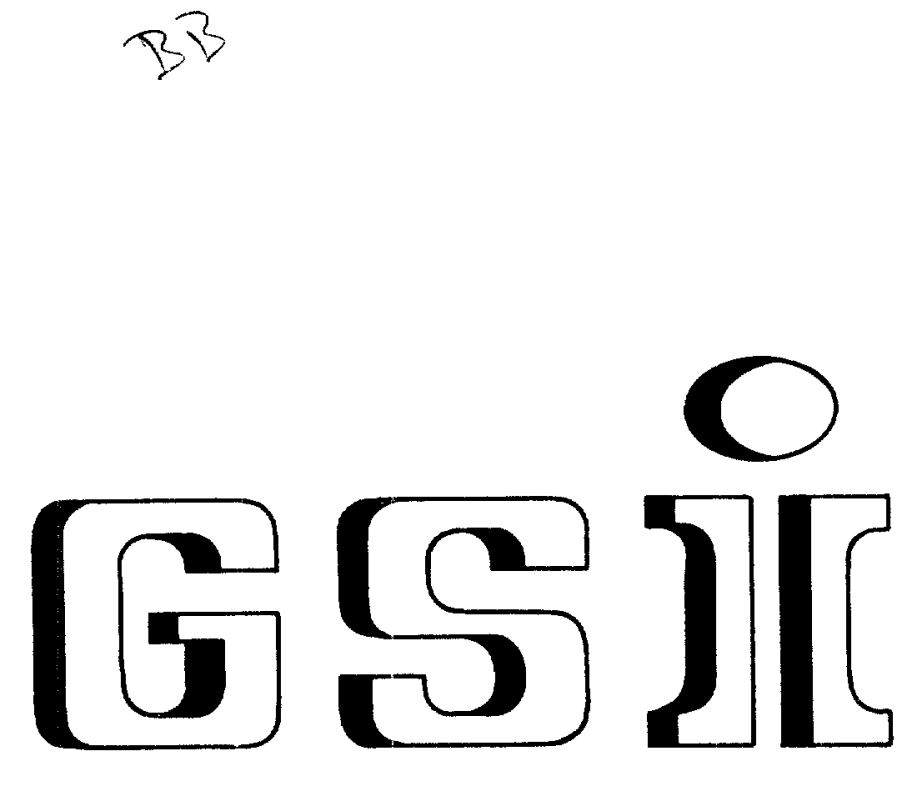

GSI-Preprint-97-53 September 1997

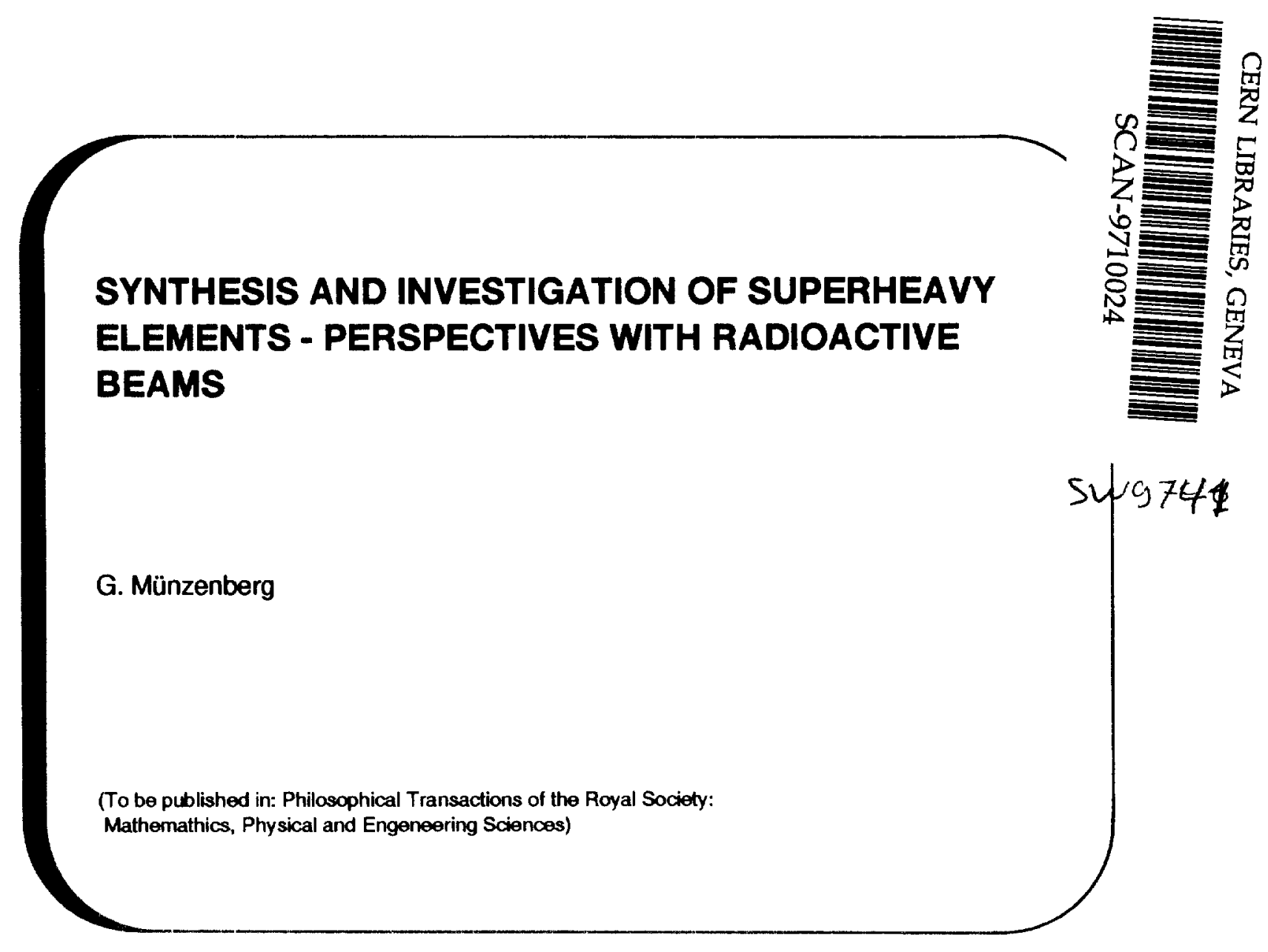

Gesellschaft für Schwerionenforschung $\mathrm{mbH}$ Planckstraße 1 - D-64291 Darmstadt - Germany Postfach 110552 - D-64220 Darmstadt • Germany 


\title{
Synthesis and investigation of superheavy elements'- perspectives with radioactive beams ${ }^{1}$ \\ Gottfried Münzenberg \\ Gesellschaft für Schwerionenforschung $\mathbf{m b H}$ \\ Planckstr. 1, D64220 Darmstadt, Germany
}

\begin{abstract}
The perspectives for the investigation of heavy and superheavy elements with intense beams of radioactive nuclei available from the new generation of secondary beam facilities in combination with modern experimental developments are the subject of this paper. The nuclear properties of the recently discovered shell nuclei centered at $Z=108$ and $N=164$ and predictions on the location of the superheavy region with improved theoretical models will be discussed.
\end{abstract}

\section{Introduction}

\subsection{Superheavy nuclei - the extension of the chemical elements}

The creation of three new elements $[1,2,3]$ with proton numbers 110,111 , and 112 close to the predicted superheavy proton shell closure $Z=114$ and the new generation of radioactive beam facilities providing accelerated secondary beams of neutron rich nuclei and new high current accelerators [4] open interesting new possibilities for superheavy element research. The discovery that the heaviest known elements belong to a region of shell stabilized, deformed nuclei $[5,6]$ centred at $\mathrm{Z}=108$ and $\mathrm{N}=164$ already located in the region of macroscopic instability [7] motivated a series of theoretical calculations with macroscopic microscopic and recently also with selfconsistent methods $[8,9]$.

The fundamental interest in heavy element research is the exploration of the limits of nuclear stability towards the heaviest nuclei and the question how many elements could exist in nature. A first answer was given by Meitner and Frisch[10] immediately after the discovery of nuclear fission by Hahn and Straßmann, when they explained the fission process in terms of the disintegration of a charged nuclear droplet. The limits for the existence of heavy nuclei are determined by the delicate balance of the Coulomb repulsion between the large number of protons and the attractive nuclear forces. Consequently spontaneous fission is the decay mode specific to the elements at the top of the nuclear table, uranium and beyond. Meitner and Frisch estimated that the periodic table would end at about element $\mathrm{Z}=100$. Detailed investigations on the fissioning nuclear droplet by Bohr and Wheeler[11] and Feenberg[12] located the upper end of the periodic table close to 110 protons.

The question arose whether shell stabilization could be strong enough to extend the nuclear table beyond the macroscopic limit into the new region of superheavy nuclei. A number of calculations performed by Mosel and Greiner[13], Fizet and Nix[14], Randrup[15], to give some important examples, predicted an

\footnotetext{
${ }^{1}$ To be published in: Philosophical Transactions of the Royal Society: Mathematics, Physical and Engeneering Sciences
} 


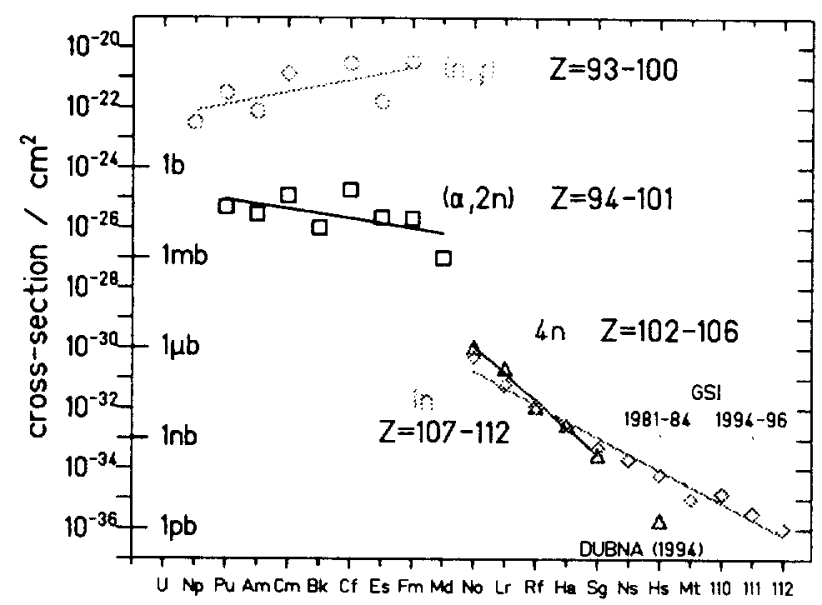

Figure 1: Synthesis reactions and production cross sections for the artificial elements beyond uranium.

island of superheavy nuclei at the double shell closure $\mathrm{Z}=114$ and $\mathrm{N}=184$, well separated from the upper end of the periodic table by a sea of fission instability. The predicted halflives ranged up to $10^{9} \mathrm{y}$, long enough for superheavy nuclei to exist in nature [14].

\subsection{Production of transuranium elements}

The first elements beyond uranium were created by breeding in nuclear reactors. By neutron capture and subesequent $\beta$-decay it is possible to climb up element by element until this path is closed by fission which occurs at fermium. All of the transactinide elements, these are the elements $\mathrm{Z}=104$ and beyond, are produced by complete fusion of heavy ions $[16,17,18,19,20]$. Fig. 1 compiles the production cross sections. For complete fusion they are far below the geometric cross section - in contrast to those for neutron capture - and drop quickly below the nanobarn level towards the heaviest elements.

Two types of reactions have been used sucessfully so far. Actinide targets close to the element to be produced were irradiated with light beams ranging from carbon to sulphur, and the fusion of lead or bismuth targets with correspondingly heavier beams ranging from calcium to zinc. This second type of reaction leads to cold compound systems[21] and has been used as the only successful route to synthesize the heaviest elements known at present. Both synthesis reactions have in common small production cross sections dropping to the order of picobarns for the heaviest elements. The respective production rates with present techniques count in atoms per day or even per week.

Upgraded and new radioactive beam facilities producing intense exotic nuclear beams with energies above the Coulomb barrier will provide neutron rich beams for heavy-element research[22, 23]. Existing laboratories such as GANIL (Caen, France), RIKEN (Tokyo, Japan) and its upgrade[24], MSU (East Lansing, USA) and its upgrade, and GSI (Darmstadt, Germany) and its upgrade are based 


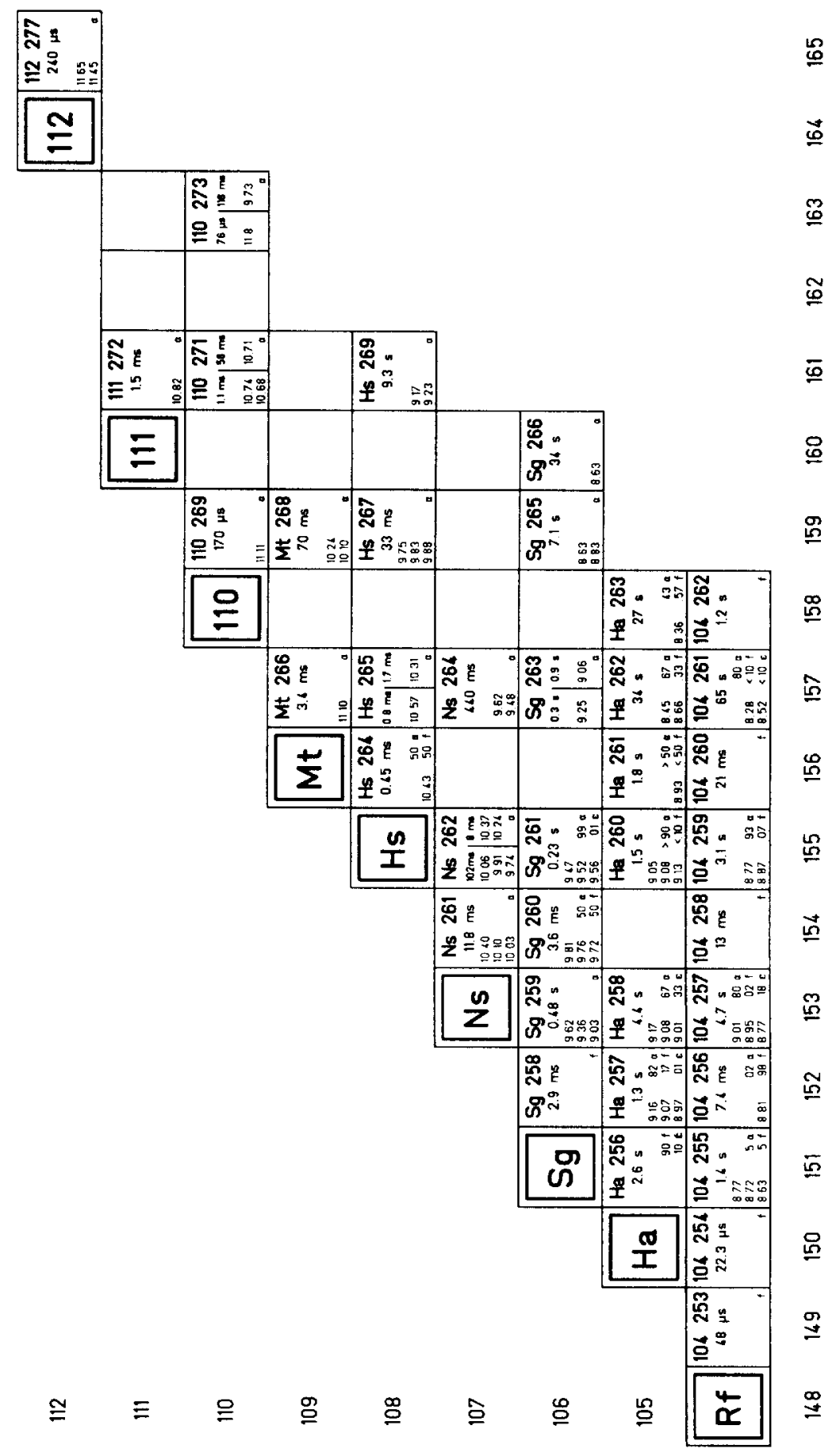

Figure 2: The upper end of the periodic table 


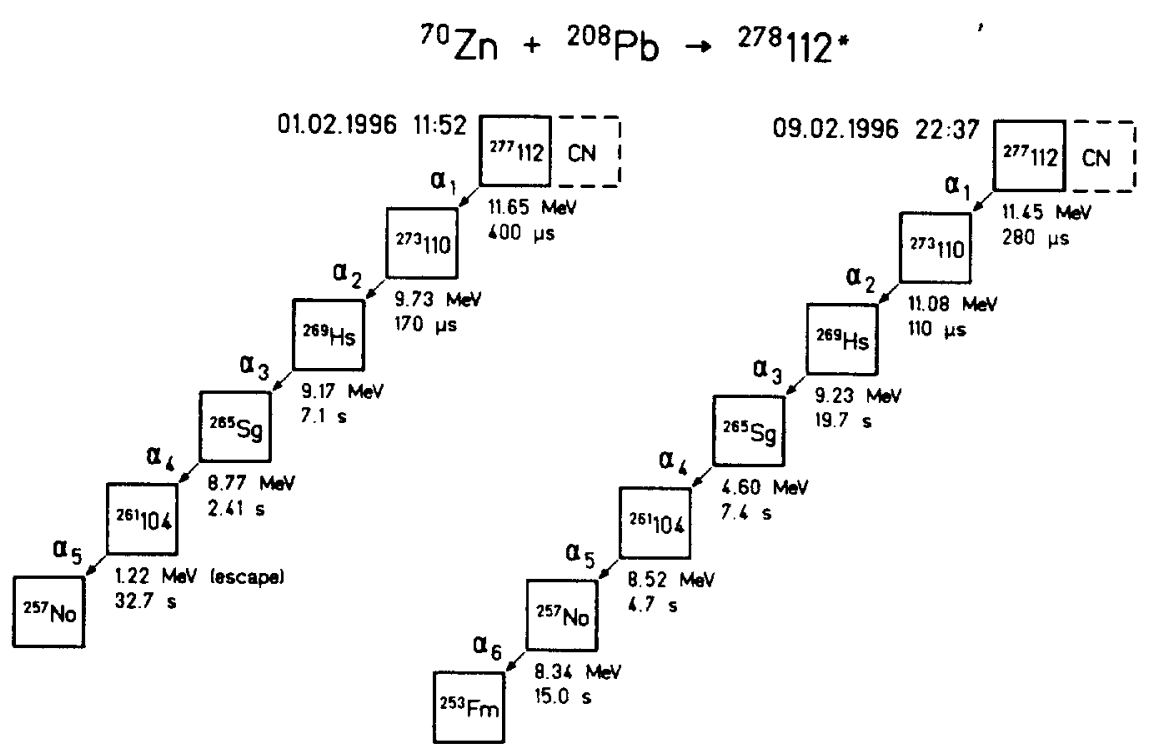

Figure 3: The decay chains observed for element 112, the heaviest element known at present.

on the fragmentation of relativistic projectile beams and are less suited for heavy element production due to the high beam energy far above the Coulomb barrier. A new generation of planned and starting facilities is based on the acceleration of radioactive beams from on-line separators to energies close to the Coulomb barrier. Those are SPIRAL of GANIL[25], EXCYT (Catania, Italy), Oak Ridge (USA), Argonne (USA)[26], INS (Tokyo, Japan) and the reactor based facilities PIAFE (Grenoble), and the Munich Project (Germany). The expected intensities are $10^{7} / \mathrm{s}$ for the running, and up to $10^{12} / \mathrm{s}$ for the projected facilities, close to $5 \cdot 10^{12} / \mathrm{s}$ used at present for heavy element synthesis. New high current accelerators for beams of stable nuclei will provide beams of up to $10^{15} / \mathrm{s}$, necessary to proceed beyond element 114 .

In the experiments with stable beams only neutron deficient isotopes of the heaviest elements could be produced. Element 112 is fairly close to the spherical proton shell, but the discovered isotope with mass 277 is still far away from the magic neutron number. Radioactive neutron rich beams will allow us to approach the spherical $N=184$ region. To profit fully from the new possibilities to synthesize heavy and superheavy elements, new reactions such as the hitherto unexplored fusion of symmetric systems (reversed fission) and the importance of isospin and shell effects need further exploration. The discovery of sufficiently longlived nuclides in the shell region interconnecting the transuranium- and the superheavy elements opens up the possibility to investigate the structure of the new species of closed shell nuclei in the transition region from deformed to spherical.

Moreover a number of new experimental developments such as in-flight sepa- 


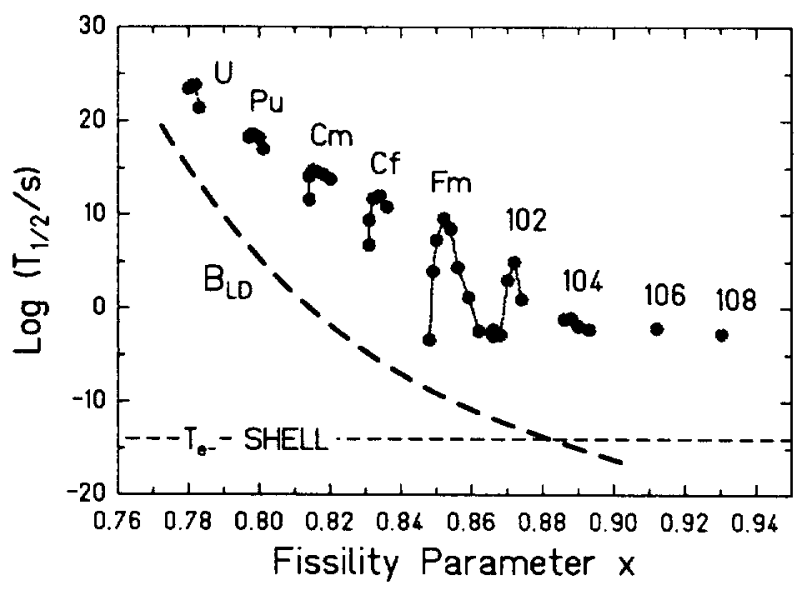

Figure 4: The partial fission halflives of the doubly even isotopes from Uranium to element 108.

ration, efficient $4 \pi$ germanium detectors, and ion traps will give new access to nuclear and atomic structure. The expected long halflives of the neutron rich species which can be produced with radioactive beams open up possibilities of chemical investigations which will be adressed briefly.

\section{Recent experimental results - our present understanding of su- perheavy nuclei}

\subsection{Nuclear properties}

Heavy element research at the very limits of nuclear stability is physics with single atoms. Safe and background free identification of individual atoms allows one to extract gross properties [27, 22] such as dominant decay modes, halfife, decay energy, and mass. Even groundstate deformations can be evaluated[19]. With the inclusion of theoretical models experimental shell correction energies, fission barriers and groundstate deformations have been obtained $[18,19]$.

In this section recent experimental results of importance for our understanding of the properties of the elements at the top of the nuclear table are summarized. More details are given in a number of reviews $[16,17,18,19,20]$. Our present knowledge on the decay properties of the heaviest elements is summarized in Fig. 2 which shows the upper part of the chart of nuclides with the observed decay modes, halflives, $\alpha$-decay energies, and branching ratios.

The name symbols are given as accepted by CNIC[28] based on the proposals of the discoverers. CNIC is the commission for nomenclature of the International Union of Pure and Applied Chemistry (ICPAC) preparing the name proposals for the plenary meeting in August 1997. It stands: Db for Dubnium $(Z=104)$, Ha for Hahnium $(Z=105)$, Sg for Seaborgium $(Z=106)$, Bh for Bohrium $(Z=107), \mathrm{Hs}$ for Hassium $(Z=108)$ and $\mathrm{Mt}$ for Meitnerium $(Z=109)$. For elements 110,111 and 112 no names are proposed up to now. Claims on naming elements 104 and 105 have not been completely settled yet, so changes cannot 


\section{$E(\mathrm{MeV})$}

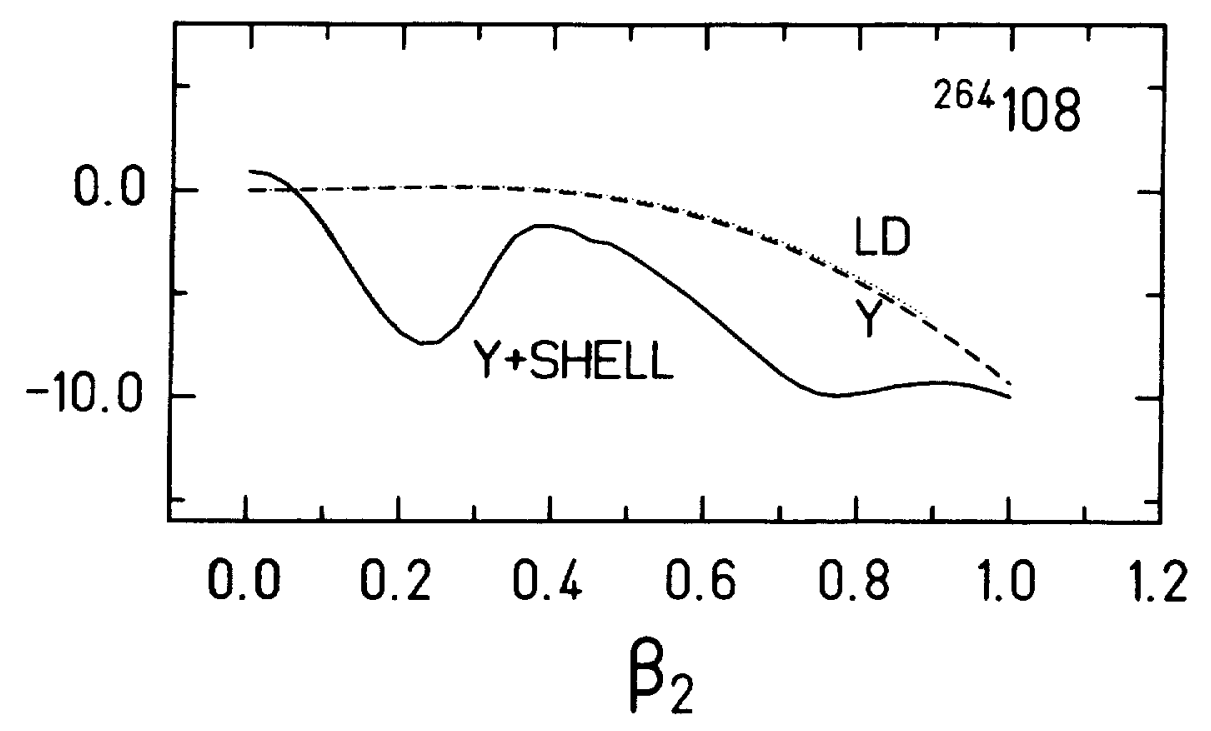

Figure 5: Calculated fission barrier for ${ }^{264} 108$, a typical deformed shell nucleus. The liquid drop (LD) barrier is indicated by the dashed line.

yet be excluded safely.

The observation of $\alpha$-decay as the prominent decay mode of element 106 indicated for the first time an enhancement of stability against spontaneous fission[29,6]. This was in contrast to the early results and extrapolations where all even-even isotopes beyond nobelium were predicted to fission with halflives microseconds or less[30]. The experimental results on the stability of element 106 against fission indicating the onset of a microscopic stabilization dramatically changed our understanding of the heaviest elements.

Element 108 was identified in the reaction ${ }^{208} \mathrm{~Pb}\left({ }^{58} \mathrm{Fe}, \mathrm{n}\right){ }^{265} 108$ on the basis of three atoms $[32,33,34]$ and confirmed in a later experiment where $75 \alpha$-decays of this isotope were observed in the same reaction[1]. The isotope ${ }^{264} 108$ is the heaviest doubly even nucleus known at present, it decays by $\alpha$-decay and spontaneous fission [34, 2]. The heaviest isotope ${ }^{267} 108$ was produced in irradiations[35] of ${ }^{238} \mathrm{U}$ with ${ }^{34} \mathrm{~S}$ and confirmed[1] when it appeared as the daughter in the decay chain of ${ }^{271} 110$. The halflives range from $0.45 \mathrm{~ms}$ for the doubly even isotope with mass 260 to $33 \mathrm{~ms}$ for the most neutron rich isotope with mass 269 .

Element 109 was first observed in irradiations of ${ }^{209} \mathrm{Bi}$ with ${ }^{58} \mathrm{Fe}$ by measuring the $\alpha$-decay chain originating from one single atom. It was assigned to the isotope with mass 266 on the basis of a careful statistical analysis of the decay sequence $[36,27]$, a result confirmed in later experiments[37, 31]. The isotope ${ }^{268} 109$ was discovered[2] in the decay chain from element 111 . The halfives of the two $\alpha$-emitting isotopes are $3.4 \mathrm{~ms}$ and $70 \mathrm{~ms}$, respectively. Presently 


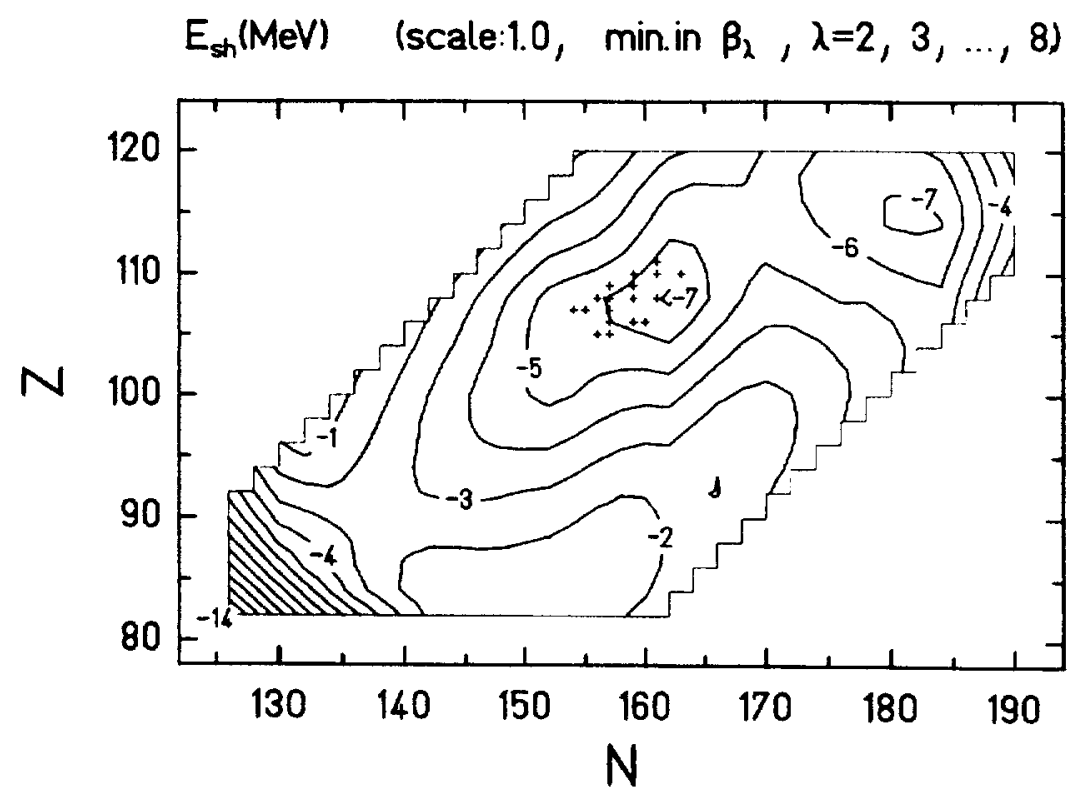

Figure 6: Shell correction energies for the heavy and superheavy elements. The known isotopes of the heaviest elements are marked by crosses.

altogether 13 atoms of element 109 have been produced, not counting those appearing in decay chains.

Element 110 was produced[1] in the reaction ${ }^{208} \mathrm{~Pb}\left({ }^{62} \mathrm{Ni}, \mathrm{n}\right)$ by the identification of the isotope ${ }^{269} 110$. The more neutron rich isotope ${ }^{271} 110$ was synthesized by using a ${ }^{64} \mathrm{Ni}$ beam. The production cross section was $15 \mathrm{pb}$ and enhanced by a factor of about three as compared to the ${ }^{62} \mathrm{Ni}$ irradiation [2]. Two more isotopes are reported, ${ }^{267} 110$, produced [39] in the irradiation of ${ }^{209} \mathrm{Bi}$ with ${ }^{59} \mathrm{Co}$ which needs confirmation as the daughter decays in the chain are not observed, and ${ }^{273} 110$ produced by irradiations[38] of ${ }^{244} \mathrm{Pu}$ with ${ }^{34} \mathrm{~S}$, a result which was confirmed when this isotope appeared in the decay chain of element 112.

Element 111 was created in the reaction ${ }^{209} \mathrm{Bi}\left({ }^{64} \mathrm{Ni}, \mathrm{n}\right)$. Altogether three $\alpha-$ chains were measured[2]. The observed isotope ${ }^{272} 111$ has a halflive of about $1.5 \mathrm{~ms}$.

The heaviest element known today with proton number 112 was discovered[3] in 1996. In an experiment of 43 days irradiating ${ }^{208} \mathrm{~Pb}$ with ${ }^{70} \mathrm{Zn}$ the two decay chains displayed in Fig. 3 were observed. They show a remarkable signature. When the chain crosses the 162 neutron isotone there appears a large gap of 1 $\mathrm{MeV}$ in the alpha decay energies accompanied by strongly enhanced halflives. This can be interpreted as the direct visibility of shell crossing.

\subsection{The new shell region beyond rutherfordium}

The most striking results of recent heavy element research are:

- the dominance of $\alpha$-decay in the heaviest elements,

- the increasing halflives for the neutron rich nuclides, 


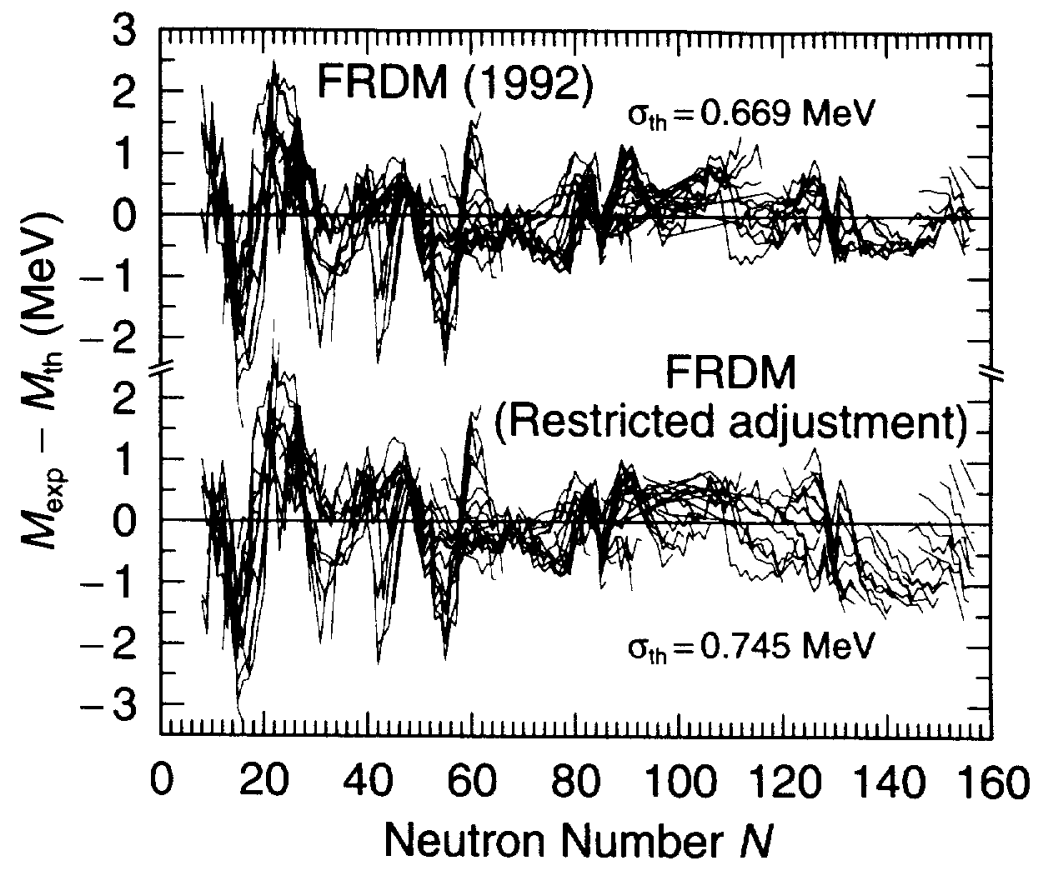

Figure 7: The differences between experimental masses and predictions from the macroscopic-microscopic model for two different parameter adjustments.

Both indicate an enhancement of nuclear stability in the region beyond rutherfordium.

Fig. 4 shows the partial fission halflives of the doubly even isotopes on a logarithmic scale plotted versus the fissility parameter, a macroscopic scaling parameter characterizing the stability of a charged nuclear droplet against fission. From uranium to nobelium halflives decrease by over more than twenty orders of magnitude, from the age of the universe down to seconds in accordance with the expectations from the nuclear liquid drop model. The enhancement of fission halflives in the isotopic chains of curium, californium, and fermium are due to a small shell effect at $N=152$. The halflife systematics change completely at element 104. Halfives become independent of the fissility parameter and stay almost constant. A comparison of the experimental data with the calculated liquid-drop halflives shows an enhancement by 15 orders of magnitude for the heaviest elements which must be attributed to microsopic stabilization.

If we define the halflife limit above which a chemical element can be accepted as the time needed for a nucleus to acquire its outer electrons [40] we arrive at $10^{-14} \mathrm{~s}$. According to this definition the nuclear chart would be terminated near element 104, but shell stabilization extends it up to the present experimental limit of $Z=112$.

Fig. 5 shows a typical shell barrier $[41,42]$ for a transactinide nucleus in the example of ${ }^{264} 108$; it is single-humped and thin. The liquid drop part of the barrier, indicated by the dashed line, is zero. The outer part of the double humped 


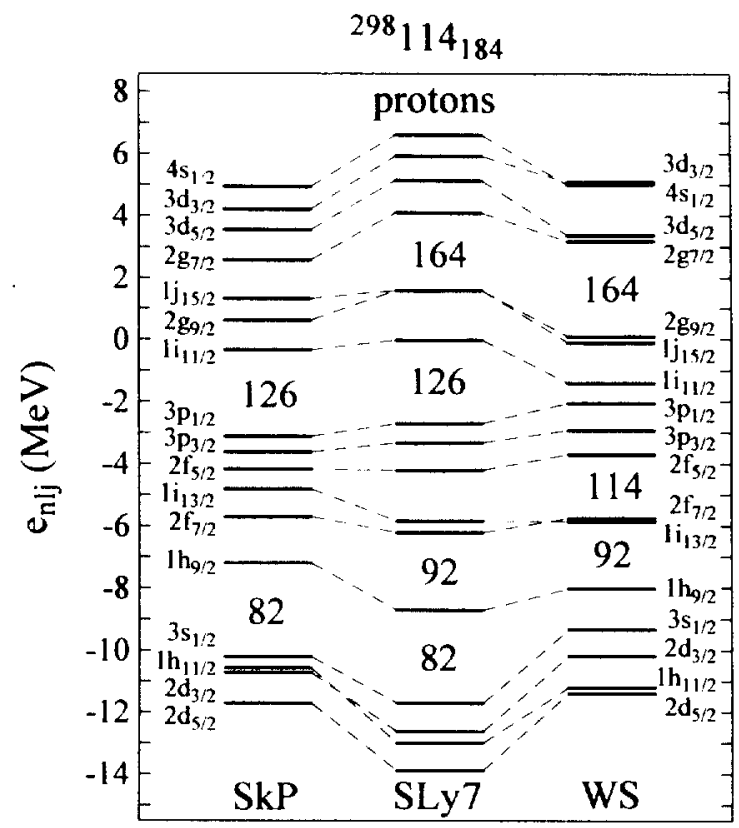

Figure 8: The proton levels and shell gaps for Hartree-Fock calculation with the SKP and SLy7 parametrizations and the Woods-Saxon single-particle potential.

barrier characteristic of the actinides, sitting on top of the macroscopic barrier at large deformation, has dropped below the groundstate with the vanishing droplet barrier and becomes ineffective.

The shell stabilization of the transactinides has its origin in a negative hexadecapole deformation in the groundstate as explained by theoretical models [ 43 , $44,45,7]$. The trend of increasing shell correction energies in the transactinide region is well reproduced by the theoretical models $[6,19,42,46]$ while the absolute values may differ by up to $1 \mathrm{MeV}$.

Fig. 6 shows the full landscape of microscopic corrections between the shell closures at lead and element 114 calculated in the macroscopic-microscopic approach with the Woods-Saxon single particle potential[46]. The shell correction energies show the steep minimum near the strong lead shell centred at ${ }^{208} \mathrm{~Pb}$, and a shallower minimum for the spherical superheavy shell closure at ${ }^{298} 114$. The new shell region of deformed nuclei centred at ${ }^{270} 108$ interconnects the transuranium elements and the spherical superheavies. The crosses mark the isotopes of the heaviest elements known today. They extend well into the new shell region. It should be noted here that the decay chains from ${ }^{277} 112$, of Fig. 3 pass close by the stability maximum. 


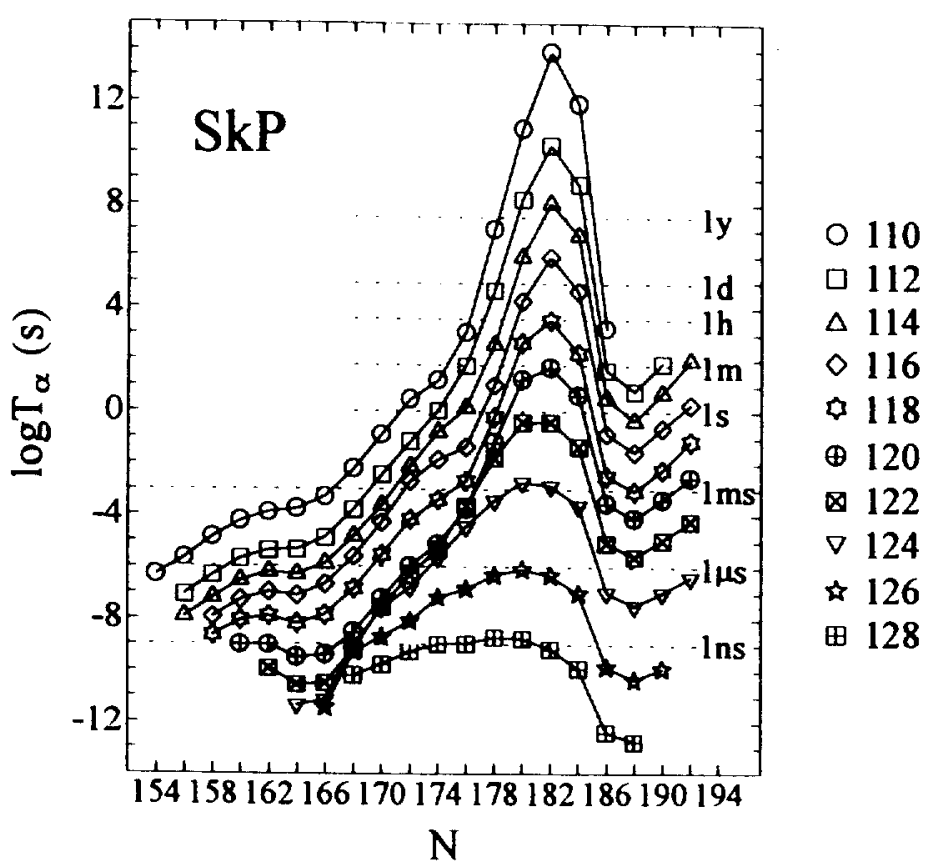

Figure 9: Partial $\alpha$-halflives for the superheavy elements $\mathrm{Z}=110$ to $\mathrm{Z}=126$ calculated with the SkP parametrization.

\subsection{New predictions}

The macroscopic-microscopic theory reproduces the groundstate properties of the heaviest elements with sufficient precision. A comparison of the experimental masses with the calculations suggests, however, some systematic deficiencies in the model for the heaviest known species[34] as shown in Fig. 7. The masses from the finite-range droplet model with microscopic corrections [47] show a trend of systematically increasing deviations from experimental data towards the heaviest elements, even more dramatic for the restricted adjustment where only masses below $\mathrm{A}=208$ have been used for the parameter fit. This discrepancy has been interpreted as a distortion of the macroscopic surface, caused by the redistribution term of the droplet model which is related to the charge density in the nuclear droplet [48].

Recently new calculations to predict the properties of the the heavy and superheavy elements have been perfomed in the selfconsistent approach using Skyrme forces or the mean field approach $[8,49]$. These calculations give different predictions for the proton shell closure at 114, 120, or 126 protons. Fig. 8 shows examples [8] for the macroscopic-microscopic approach with the Woods-Saxon Potential (WS), and self consistent Hartree Fock calculations with Skyrme forces SKP and SLy7.

There are a number of halflife predictions for the heavy and superheavy elements, mostly from the macroscopic-microscopic theory for fission and $\alpha$-decay 


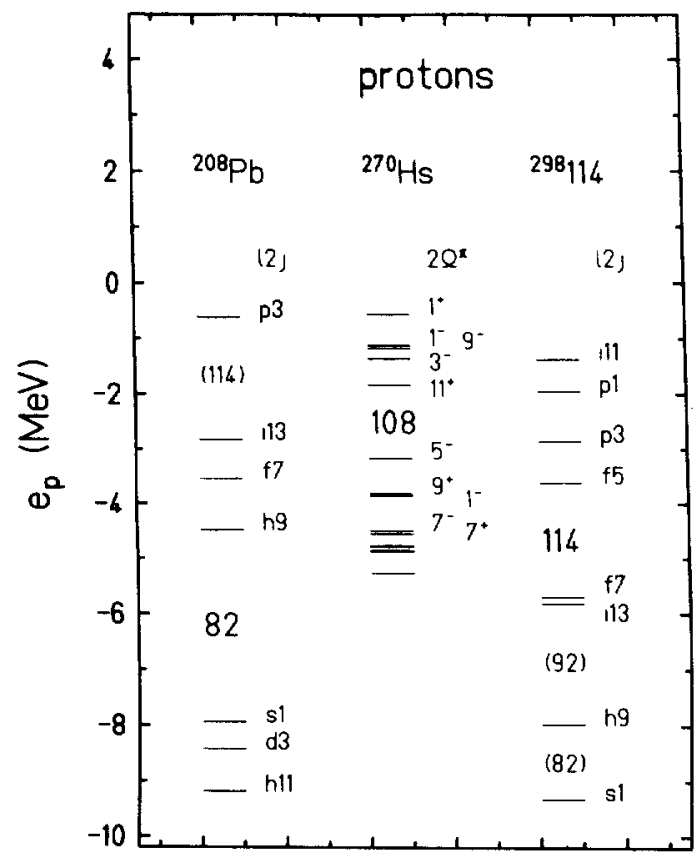

Figure 10: Proton levels for ${ }^{208} \mathrm{~Pb}$ compared to deformed and spherical superheavy nuclei $[50]$.

$[42,8]$ and also including $\beta$-decay[61]. A halflife prediction from a self-consistent calculation[8] using the Skyrme interaction SKP for the doubly even isotopes of the superheavy elements is displayed in Fig. 9. For the known isotopes the prediction agrees with the experimental data.

The heavy nuclei are predicted to be $\alpha$-emitters [42]. The longest-lived isotopes near $\mathrm{N}=184$ have partial $\alpha$ halflives exceeding $10^{6}$ years. The hexadecapole stabilized region with maximum halflives of $10^{6}$ years forms a bridge between the upper end of the periodic table and the double shell closure where halflives of the elements above $\mathrm{Z}=124$ never drop below the order of $\mu$ s allowing one to approach the superheavy-region stepwise with in-flight techniques, offering a rich field of neutron rich nuclides for investigation, synthesized using radioactive beams - and targets.

Halflives of odd nuclei are longer due to the hindrance of fission and $\alpha$-decay. Note that the calculations do not include $\beta$-decay, which will shorten the halflives of the longlived isotopes. For the heavy elements near $Z=112$ the valley of $\beta$-stability comes close to $N=184$ so $\beta$-decay of the longlived species is excluded.

\section{Perspectives for nuclear structure studies with radioactive beams}

The neutron rich, deformed shell nuclei bevond $\mathrm{N}=162$ covering the region between the transuranium elements and the spherical superheavy shell closure 


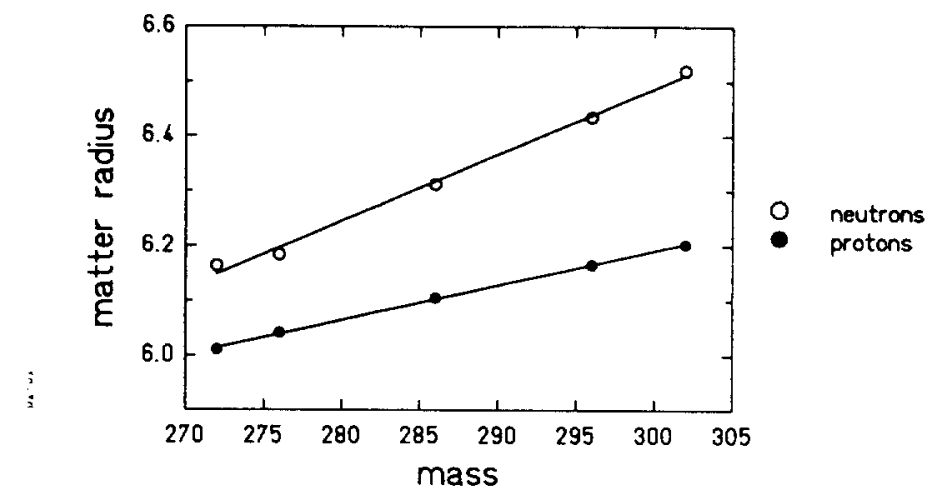

Figure 11: Calculated neutron and proton rms radii fro the isotopic chain of element 112 plotted versus the mass number [53].

offer a rich field for nuclear structure research with radioactive beams to investigate the behaviour of complex nuclear systems with balanced nuclear and Coulomb forces. The fact that the expected halflives in this region exceed the order of seconds will open up this region for the continuation of chemical investigations and the application of new experimental techniques such as ion traps for direct mass measurements and laser spectroscopy to investigate nuclear and atomic properties.

Moreover for the heaviest elements beyond 112 the decay chains end in neutron rich isotopes which are unknown as yet, so the parent-daughter correlation method is not applicable for identification. The combination of heavy-element synthesis with stable and with neutron rich radioactive beams would access this region directly on the one hand and by overshooting on the other hand and thus be compatible. Similar arguments hold for the nuclear structure investigations discussed in the following section.

\subsection{Nuclear structure at large proton numbers and beyond $Z=162$}

The nuclei beyond rutherfordium represent the species of nuclei beyond macroscopic stability. As the underlying macroscopic force is ineffective already small shell gaps may create new shapes, so that a rich coexistence of nuclear deformations in all degrees of freedom may be observed. It has been debated whether there exists a spherical shell [51] at $\mathrm{N}=164$. In fact the two decay chains of element 112 which are slightly different might indicate such an effect. The second chain shows the largest energy step when crossing $N=162$, the largest step in the first chain already appears in the first generation[3] when the decay sequence crosses $N=164$. A final answer to this question certainly needs further data along the isotones in the vicinity of the respective neutron numbers.

The elements at the upper end of the nuclear chart are the most complex nuclear systems. They have large level densities and high angular momenta. The complex structure of the heaviest elements is already visible in our data showing a number of isomers. High- and low spin orbits are coming close together and offer a rich field of interesting spectroscopy. Fig. 10 shows the 
proton levels for the deformed and the spherical shell gaps for ${ }^{270} 108$ and ${ }^{298} 114$ calculated with the Woods-Saxon single particle potential [50]. The figure shows that both gaps are about $2 \mathrm{MeV}$, less than that of the lead shell which amounts to more than $3 \mathrm{MeV}$. The level densities near the deformed shell are much higher than those around the spherical shell where low densities indicate an extended region of large shell corrections above $Z=114$. This is visible in the map of shell correction energies (Fig. 6).

Because of their large volume the heaviest elements are the most appropriate objects to investigate bulk nuclear matter. Another interesting aspect is the study of nuclear structure under the influence of a strong Coulomb field. Selfconsistent calculations $[8,52,53]$ on the charge distribution in the superheavy nuclei show that the strong Coulomb forces in the heaviest elements create an enhanced charge density at the nuclear surface, affecting the high-spin orbits which are sensitive to the nuclear surface as they are pushed out from the centre of the nucleus due to the angular momentum barrier. The spin-orbit splitting at large mass values and its behaviour for the large diffuseness at the nuclear surface will show up in the level structure.

A mapping of the surface sensitive orbitals, such as isomeric states at high spin over an extended $\mathrm{Z}$ range along isotonic chains will help us to learn about the diffuseness of the proton surface. The systematic investigation of large isotopic chains will help us to understand the neutron-proton interaction at the surface, as the neutrons will follow the protons. These questions are related to the investigation of nuclear skins for the lighter nuclei. Fig. 11 shows the charge and neutron radii of the $\mathrm{Z}=110$ isotopic chain [53] obtained from a HF-Bogoliubov calculation. Though the known isotope of element 112 is close to the proton dripline it already has a large neutron excess and forms a thin neutron skin growing to a thickness of $0.3 \mathrm{fm}$ for the isotopes close to $\mathrm{N}=190$, close to $\beta$-stability. Such a skin thickness is comparable to that observed for extremely neutron-rich light nuclei, as e. g. sodium. For increasing neutron number charge radii increase due to pn coupling.

The question of where the spherical shell closures are located with respect to the superheavy elements, can only be answered in experiments proceeding to continuously heavier elements. Valuable nuclear structure information even on the nuclei at the very top needs careful investigation of the lighter species which can be produced in sizeable quantities, as demonstrated in a recent experiment [54] where more than a thousand atoms of ${ }^{257} 104$ were produced and detailed structure information was obtained from $\alpha$-decay.

The neutron rich species are predominantly important to extract structure information for the elements at the top of the nuclear table from the structure along isotones. As the heavy elements at the top produced with stable beams tend to be increasingly neutron rich, the isotones in the lighter nuclei are only accessible with neutron rich radioactive beams. Moreover $\alpha$-chains follow favoured transitions between similar spin states. Consequently $\alpha$-chains will populate nuclear states which may be different from those populated directly in the fusion process, as observed for element 108 in the element 110 decay chains [1]. Knowledge of the structure of the nuclei at the ends of the chains reveals some of the structure of the mother nuclei, but the full structure information about 


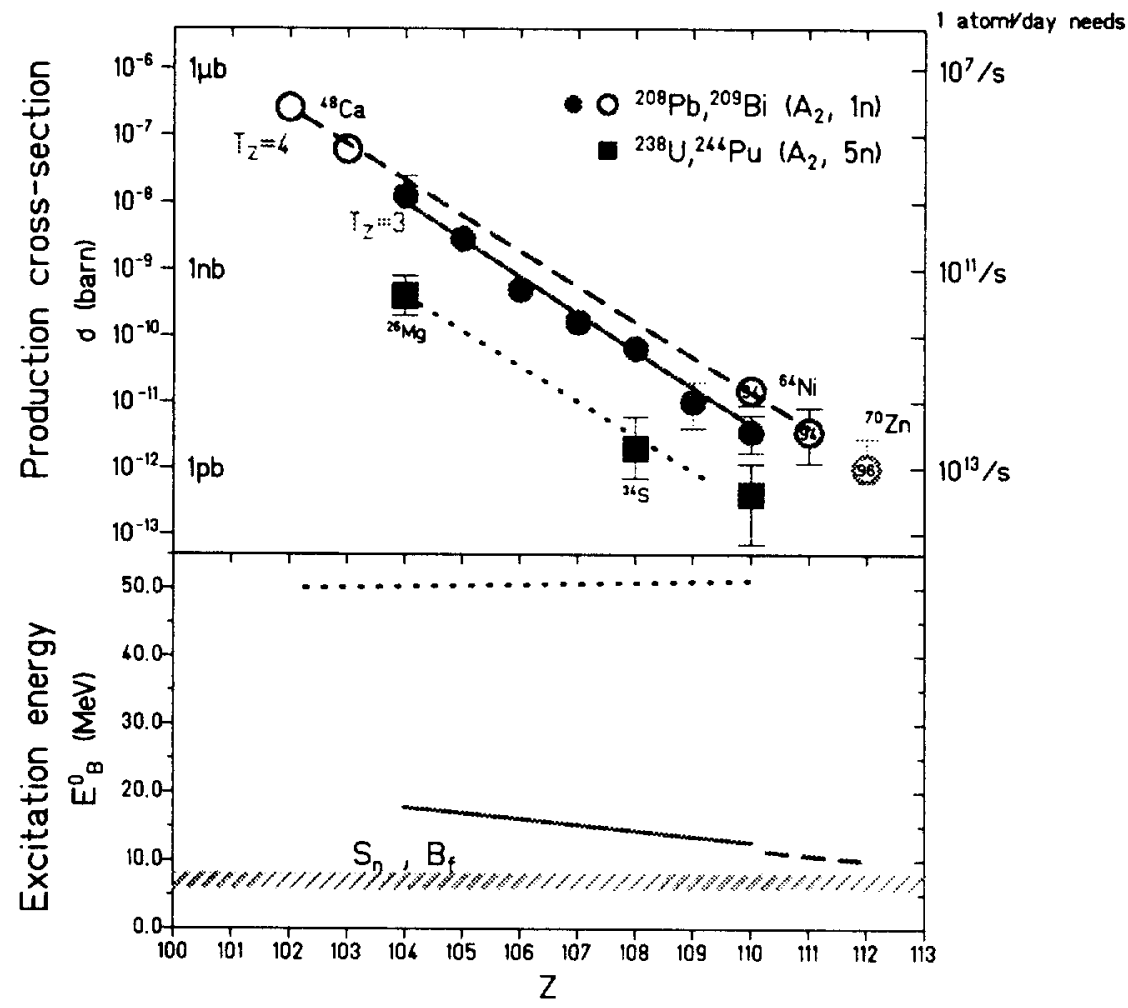

Figure 12: Production cross sections for the heaviest elements plotted versus the proton number (upper panel) and excitation energies at the maximum production cross section (lower panel). The beam intensities needed for the production of one atom per week are given on the lefthand scale.

a nucleus is obtained by its study in direct production.

\subsection{Extension of the deformed region and the end of the r-process}

One interesting question is how far the deformed island extends and how stability continues towards the spherical neutron shell in the elements around fermium, and how this affects nuclear halflives. This question is related to the end of the r-process path. The neutron rich region around fermium is of particular interest. In that region new fission modes, symmetric and bimodal fission, were discovered [16]. The fission halflives of fermium drop extremely quickly towards 158 neutrons and fall even below that of the transactinides. This was explained by a new fission path following compact shapes, and supported experimentally by a narrow symmetric mass distribution accompanied by a high total kinetic energy of the fragments. The distributions are influenced by the fragment shell structure of the the deformed shells along the fission path. This effect might affect fission halflives strongly and totally compensate for shell stabilization. 


\section{Production of heavy and superheavy elements}

\subsection{Fusion of heavy systems}

Fig. 12 displays the cross sections for the production of the heaviest elements in terms of the proton number of the final residue. The heaviest known elements were produced by cold, heavy ion fusion[21]. To produce elements with even proton number or odd proton number lead or bismuth is irradiated with beams of ${ }^{48} \mathrm{Ca},{ }^{50} \mathrm{Ti},{ }^{54} \mathrm{Cr},{ }^{58} \mathrm{Fe},{ }^{62,64} \mathrm{Ni}$, or ${ }^{70} \mathrm{Zn}$. The cross sections drop from the order of $\mu \mathrm{b}$ for nobelium to one $\mathrm{pb}$ for element 112 and show a dependence on the projectile isospin. An increase of the isopsin by 1 unit enhances the production cross section by a factor of about five. The dots refer to $\mathrm{T}_{Z}=3$, circles to $\mathrm{T}_{Z}=4$. The excitation energies of the compound system for the maximum production cross section are around $10 \mathrm{MeV}$ for element 112 (Fig. 12, lower panel). For such cold systems the evaporation of one neutron is already sufficient to dissipate the excitation energy of the compound nucleus. The isospin dependence of the production cross section can be explained most simply in terms of a decrease in excitation energy of the compound system at the barrier for the more neutron rich projectiles.

The production cross sections with actinide targets, ${ }^{238} \mathrm{U}$ and ${ }^{244} \mathrm{Pu}$, in $5 \mathrm{n}$ and $4 \mathrm{n}$ reactions are indicated with filled squares. They are more than one order of magnitude below the cold fusion cross sections. The heaviest element created with this type of reaction[38] is element 110 .

The production of a heavy nucleus is a two step process. In the first step the projectile has to penetrate the Coulomb - or more precisely - fusion barrier, then the nuclear forces drive the dinuclear system from the contact configuration inside the fission saddle, which prevents the system from immediate disintegration. In general the energy necessary for the projectile to overcome the Coulomb barrier is higher than the energy needed for the excess of binding energy in the heavy nucleus, the compound system is excited. The excitation energy is dissipated by neutron evaporation and $\gamma$ emission. For the heaviest nuclei there is a strong fission competition, which is more than three orders-of-magnitude higher than the probability for neutron emission.

The production cross section for the fusion-evaporation reaction is:

$$
\sigma=\pi \star^{2} \Sigma(2 l+1) \cdot T_{l} \cdot w_{x}
$$

where $l$ is the angular moementum of the colliding system. The angular momentum limit for the fissile heavy elements is below $15 \hbar$, corresponding to about $10 \mathrm{mb}$ cross section. Heavy elements are produced in central collisions. $T_{l}$ is the angular momentum dependent transmission through the fusion barrier, and $w_{x}$ the survival probability of the compound system after the evaporation of $\mathrm{x}$ neutrons. It can to a first approximation be written as

$$
w_{x}=\left(\Gamma_{n} / \Gamma_{f}\right)^{x}
$$

with $\Gamma_{n} / \Gamma_{f}$ characterizing the neutron-to-fission width. The idea of the cold fusion can immediately be derived from this relation: for the heaviest elements 


\begin{tabular}{lrrr}
\hline & & & \\
SPIRAL & ${ }^{24} \mathrm{Ne}$ & $7 \cdot 10^{7}$ & $\mathrm{~s}^{-1}$ \\
& ${ }^{44} \mathrm{Ar}$ & $5 \cdot 10^{7}$ & $\mathrm{~s}^{-1}$ \\
PIAFE & ${ }^{78} \mathrm{Zn}$ & $10^{8}$ & $\mathrm{~s}^{-1}$ \\
& ${ }^{84} \mathrm{Ge}$ & $2 \cdot 10^{8}$ & $\mathrm{~s}^{-1}$ \\
& ${ }^{94} \mathrm{Kr}$ & $2 \cdot 10^{9}$ & $\mathrm{~s}^{-1}$ \\
\hline
\end{tabular}

Table 1: Expected Intensities for neutron rich radioactive beams

which are most fissile, the survival probability is expected to be highest if the system is formed cold and only one neutron is evaporated. This is shown by the data.

The small cross sections for the production of the heavy elements cannot be explained by an infinitely small survival probability of the compound system. Models were developed to explain the losses in the entrance channel. The extrapush model developed for the fusion of massive nuclei predicts an enhancement of the fusion barrier for increasingly heavier systems due to dynamic losses in the dinuclear system on its way to fusion [55]. The small cross sections at the conventional fusion barrier can be explained in terms of an upshift of the barrier $[56,57]$.

Our experimental observation for the heavy elements shows the opposite behaviour. The excitation energies decrease towards the heavier elements: The production of element 112 is the coldest heavy-ion fusion ever observed. The macroscopic dynamics of the extra-push concept do not seem appropriate to describe the extremely cold production of closed shell nuclei[18].

First attempts to explain qualitatively the influence of microscopic effects in the entrance channel on the production of heavy and superheavy elements have been made with the fragmentation theory[59]. This model predicts that the cold fusion of closed shell nuclei, e. g. the use of ${ }^{208} \mathrm{~Pb}$ and corresponding projectiles is particularly favourable. Recently calculations in the framework of the macrosopic-microscopic approach showed that the entrance channel fragment shell gaps are preserved far inside the contact point[61].

New concepts including microscopic effects in the entrance and exit channels are being developed. The diffusion model uses a microscopic-macroscopic mode with temperature dependent shell corrections to create a fusion pocket, the depth of which is determined by the excitation energy of the system on its way to fusion[62]. The dinuclear system (DNS) concept assumes that fusion is achieved by transferring the nucleons shell by shell to build-up the mononuclear system[63]. Both models stress the importance of microscopic effects. The losses in the fusion channel are explained by diffusion into the the fission valley. The influence of microscopic effects in the compound nucleus to enhance the survival in the deexcitation process has been investigated, but without positive result [56]. 


\subsection{New possibilities with radioactive beams}

The problem to create superheavy elements with stable beams is that one can never reach the $\mathrm{N}=184$ neutron shell closure in the vicinity of $\mathrm{Z}=114$. One idea, besides the use of neutron rich exotic beams, is to profit from the increasing neutron excess in the heavier projectiles and to overshoot the proton number so that the neutron shell is crossed in the decay chains as has been achieved for element 112. The beam intensities needed for the production of one atom per week are indicated in Fig. 12. If the cross section systematics remains valid, the region beyond element 114 needs beam intensities in excess of $10^{14} \mathrm{~s}^{-1}$. These intensities can be provided by the next generation-heavy-ion accelerator at GSI.

First experiments on heavy-ion fusion with radioactive beams have already been started. Systems like ${ }^{27,29,31} \mathrm{Al}+{ }^{197} \mathrm{Au}$ and ${ }^{9,10,11} \mathrm{Be}+{ }^{209} \mathrm{Bi}$ were investigated. These pioneering experiments suffer from the quality of the data which is partly due to weak beam intensities, so it is difficult to draw final conclusions. Nevertheless new effects such as fusion enhancement with neutron rich species seem to show up[67].

Table 1 shows the expected secondary beam intensities for two examples, SPIRAL as a heavy-ion based facility under construction and PIAFE, as a reactor based project. They are of the order of $10^{8} / \mathrm{s}$. The fission fragment beams from the reactor facility are fairly neutron rich. For the next generation facilities, such as the Munich reactor project and spallation facilities with $100 \mu \mathrm{A}$ proton beams of $1 \mathrm{GeV}$ intensities may be increased by three to four orders of magnitude [65].

The extrapolation of the production rates for new elements beyond $\mathrm{Z}=112$ is difficult as the maximum of the production cross section approaches two critical values: neutron binding energy and the fission barrier of the compound nucleus. If the emission of one neutron is necessary to catch the dinuclear system inside the fission barrier, the position of the excitation function is fixed at its lower end by the neutron binding energy. Going below it means that there is no dynamics left for the fusion process and no space for the neutron to evaporate. Cross sections beyond $Z=114$ may decrease faster than expected from the systematics of Fig. 12.

Similar arguments hold for the isospin enhancement to extrapolate the coldfusion cross sections with neutron rich radioactive beams. On our present understanding isospin enhancement is only expected if there is enough room left for cooling the compound system e. g. for cold fusion in the heavy actinides. In the actinide based fusion large effects might be expected as the compound systems accessible with stable beams are produced with high excitation energy (Fig. 12). Radiative capture, though observed for lighter systems[66] has never been observed in the production of heavy elements though several attempts have been made.

The domain of the neutron rich beams will be beyond $\mathrm{N}=162$ in the actinide region below or up to element 104 where isospin enhancement should be effective and cross sections are still in the nanobarn region. Hot fusion with actinide targets has enough room left for cooling and might be extremely attractive for 


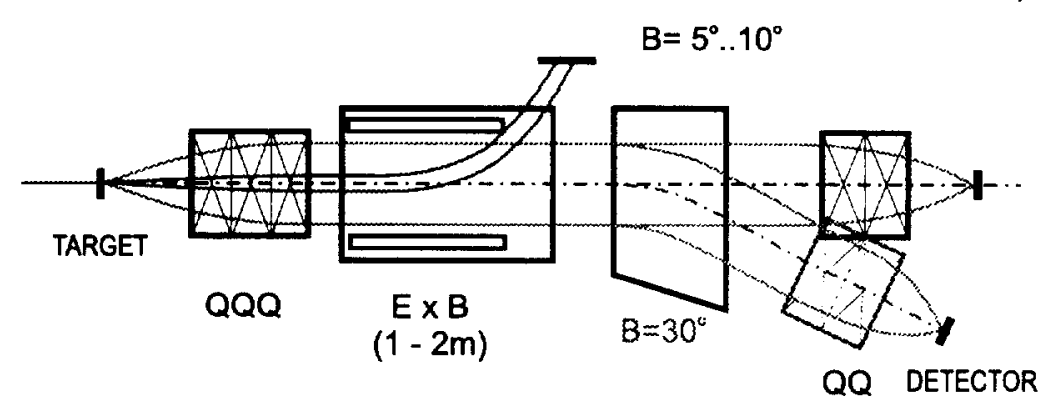

Figure 13: Schematic view of a recoil separator for applications with radioactive beams.

light beams such as neon or manganese plus curium to access elements 106 and 108 , for example.

New ways of fusion need investigation. Fragmentation theory predicts three dominant paths to heavy elements [60]: the cold fusion with lead targets, showing a deep valley, the actinide fusion, indicated by a weak valley, and in addition the symmetric fusion - or inverse fission - as yet unexplored. First experiments on this reaction have been made $[64]$ in the investigation of ${ }^{86} \mathrm{Kr}$ on ${ }^{130,136} \mathrm{Xe}$. The data could be explained in the framework of the statistical model. No upshift of the fusion barrier[58] was found.

\section{Nuclear transfer and reaction studies}

Production of heavy elements by multinucleon transfer has been investigated in an number of experiments, they were the only tool to access neutron rich actinides. Examples are the identification of ${ }^{261,262} \mathrm{Lr}$ and ${ }^{262} \mathrm{No}$ in the reactions of ${ }^{18} \mathrm{O}$ and ${ }^{24} \mathrm{Ne}$ with ${ }^{264} \mathrm{Es}$. With the availability of neutron rich beams one certainly would come further[68] to the neutron rich side.

The real power of first generation secondary beam facilities will be reaction studies. Radioactive beams extend our possibilities to investigate the importance of isospin and shell effects on fusion and transfer as they offer a rich choice of nuclides. Specifically nuclear transfer as the doorway to fusion needs further attention. New experimental tools such as in-beam $\gamma$-spectroscopy with $4 \pi$ germanium arrays would allow detailed studies and direct observation of the reaction channels not possible in the experiments up to now which were performed with nuclear chemistry.

\section{Technical developments}

\subsection{Chemistry of the transactinides}

The investigation of the neutron rich, longlived heavy elements produced with secondary beams needs the development of sensitive separation- and detection techniques for longlived species. The classical method of identification of an 
element - provided one can produce isotopes with sufficiently long halflife - is chemical separation.

Elements with haflives exceeding $1 \mathrm{~s}$ are accessible to chemical investigation. A key question is the end of the Mendelejev systematics of the periodic table. The periodic table is understood in terms of successively filling electron shells when proceeding to heavier elements. For the heavy nuclei however the Coulomb forces of the nuclear core are so strong that the inner electrons become relativistic. Because the chemical behaviour of a chemical element strongly depends on the electronic configuration, these effects may influence the chemical properties. Such influence was observed in the chemical behaviour of elements 105 and 106 , respectively[69]. The chemistry is based essentially on the study of the chemical behaviour of single atoms in volatile compounds by gas chromatography, or in aqueous solution by liquid chromatography. For these reasons chemistry is not an appropriate tool to discover hitherto unknown elements as their chemical properties are not predictable any more. On the contrary chemistry has to rely on physical methods to identify a specific element in the chemical processing.

\subsection{New in-flight techniques and detectors}

In-flight separation is the now established method for heavy element research[70]. The heavy fusion products recoiling from the target are separated in-flight with their full energy. The separation is fast. Separation times correspond to the flight time through the separator which amounts to microseconds. Moreover it is independent of chemical properties and efficient. With the recoil separator SHIP $[72,71]$ detection efficiencies for the heaviest elements are of the order of $50 \%$.

SHIP is a two-stage velocity filter designed for heavy projectiles $(M \geq 40)$ and high primary beam intensities of up to $10^{13} / \mathrm{s}$ to detect recoils with production cross sections far below nanobarns. Separators for secondary-beam application have different requirements. Most of the facilities will use light beams and have much smaller intensities. Therefore a compact separator with large acceptance is most appropriate. A large-aperture quadrupole doublet at the entrance to collect the recoils with large efficiency and one velocity filter combined with a dipole magnet is needed (Fig. 13). The dipole magnet has a variable deflection angle to be operated without charge dispersion at small angle or with increased dispersion in combination with the filter as recoil mass separator of moderate resolution.

The separated recoils are implanted into position sensitive silicon detectors with their full energy, and identified by the $\alpha$-decays following their imlantation, observed at the position of implantation[73]. Following the decay sequences to known transitions they are identified unambiguously. With the example of element 109 it has been shown that this method was successful in identifying a new element on the decay of one single atom[27].

This technique works best for sequences of shortlived species as they have the highest statistical significance. For longlived isotopes at or close to closed shells there are two strategies: as already discussed, the first is to overshoot this region. The decay chain will start at a shortlived isotope and run into the 
longlived region. Then the respective detector position can be observęd off-line until the chain continues.

To go directly into the longlived region needs improved detection techniques permitting one to identify the recoil itself. As proton emission is not observed for the producion of the heaviest elements, a determination of the mass number of the implanted recoil would be sufficient for identification. This can be achieved by time-of-flight and energy measurement. Silicon detectors have insufficient energy resolution due to the large pulse height defect for the heavy and slow fragments[27]. The recently developed bolometric detectors have been shown to provide energy resolution[74] of the order of $10^{-3}$ sufficient for mass identification in the $M=300$ region. These detectors work at liquid helium temperature and measure the temperature increase induced by the heat deposited in a crystal. They can also detect $\alpha$-decay with a resolution of better than $50 \mathrm{keV}$.

\subsection{Trapping and laser techniques}

The combination of ion traps with recoil separators will open up new possibilities such as high precision mass measurements and laser spectroscopy to determine deformations or ionization potentials. An example is the SPIG ion guide[75] coupled to a gasfilled recoil separator. A combination of an RFQ ion guide to catch the recoils, a cooler trap and a precision trap is being developed[76] to operate in combination with SHIP. It would be interesting to investigate how the charge distribution in the nuclei would show up in the isotope shifts.

\section{7 conclusion}

Recent heavy element research led to the discovery of deformed, shell stabilized nuclei creating an extended region of enhanced stability which connects the transuranium region to the magic proton number 114 , already closely approached with the discovery of element 112 . While new elements beyond $Z$ $=114$ to our present knowledge will only be acessible with intense beams deliverable from high-current accelerators, the region beond $\mathrm{N}=162$, accessible with neutron rich beams, will offer a rich field for nuclear structure studies with new phenomena expected. The long halflives will allow the application of new methods for high precision studies of nuclear and atomic properties, and chemistry.

\section{Acknowledgement}

I gratefully acknowledge fruitful discussions with P. Armbruster, S. Hofmann, H. Lenske, P. Möller and A. Sobiczewski. I thank E. Pfeng for arranging the manuscript in its final form. 


\section{References}

[1] Hofmann, S., Ninov V., Heßberger, F. P., Armbruster, P. Folger, H., Münzenberg, G., Schött, H. J., Popeko, A., Yeremin, A., Anreyev, A., Saro, S., Janik, R., Leino, M. E.: Z. Phys. A350 (1995) 277

[2] Hofmann, S., Ninov V., Heßberger, F. P., Armbruster, P. Folger, H., Münzenberg, G., Schött, H. J., Popeko, A., Yeremin, A., Anreyev, A., Saro, S., Janik, R., Leino, M. E.: Z. Phys. A350 (1995) 281

[3] Hofmann, S., Ninov V., Heßberger, F. P., Armbruster, P. Folger, H., Münzenberg, G., Schött, H. J., Popeko, A., Yeremin, A., Saro, S., Janik, R., Leino, M. E.: Z. Phys. A354 (1995) 229

[4] Münzenberg, G., The Int. Symposium on Nuclear and Particle Physics with High Intensity Proton Accelerators, Tokyo, Japan 1996

[5] Armbruster, P., From Nuclei to Stars, Proc. 91st Corso soc. Italiana di Fisica, Bologna, Italy, North-Holland, Amsterdam, 222-249(1985)

[6] Münzenberg, G., Hofmann, S., Folger, H., Heßberger, F. P., Keller, J. G. Poppensieker, K. Quint. B., Reisdorf, W., Schmidt., K. H., Schött, H. J., Armbruster, P., Leino, M. E., Hingmann, R.: Z. Phys. A322, $227-235$ (1985)

[7] Patyk, Z., Sobiczewski A., Nucl. Phys. A533, 132(1991)

[8] Cwiok, S., Dobaczewski, J., Heenen, P.hH., Magierski, P., Nazarewicz, W.: Nucl. Phys. A611, 211 (1996)

[9] Rutz, K., Bender, M. Bürvenich, T., Schilling, T., Reinhard, P.- G., Maruhn, J. A., Greiner W., to be published

[10] Meitner, L., Frisch, O.: Nature 143, 239-240 (1939)

[11] Bohr, N., Wheeler, J. A.: Phys. Rev. 56, 426-450 (1939)

[12] Feenberg, E.: Phys. Rev. 55, 504-505 (1939)

[13] Mosel, U., Greiner, W.: Z. Phys. 222, 261-282 (1969)

[14] Fizet, E. O. Nix, J. R.: Nucl Phys. A193, 647-671 (1972)

[15] Randrup, J., Larsson, S. E., Möller, P., Sobiczewski, A., Lukasiak, A.: Phys. Scr. 10A, 60-64 (1974)

[16] Seaborg, G. T., Loveland, W. D.: "The Elements beyond Uranium", John Wiley and Sons, Inc. New York (1990)

[17] Armbruster, P.: Ann. Rev. Nucl. Sci. 25, 135-94 (19825)

[18] Münzenberg, G.: Rep. Prog. Phys. 51, 57-104 (1988) 
[19] Münzenberg, G.: Radiochimica Acta 70/71 193 (1995)

[20] Hofmann, S.: Rep. Prog. Phys. to be published

[21] Oganessian, Y. Ts.: Lecture Notes in Physics 33, 221- 252 (1974)

[22] Geissel, H., Münzenberg, G. Riisager, K.: Ann. Rev. Nucl. Part. Sci. $45,163-203(1995)$

[23] Proc. Fourth Int. Conf. on Radioactive Nuclear Beams, Omiya, Japan, 1996 Eds. S. Kubono, T. Kobayashi, I. Tanihata: Nucl. Phys. A616 (1997)

[24] The RIKEN - upgrade, Int. rep. RIKEN, Tokyo, Japan (1994)

[25] The SPIRAL project, Int. Rep. GANIL, Caen, France (1994)

[26] The Argonne Radioactive beam Project, Int. Rep. Argonne Natl. Lab., Argonne, USA (1995)

[27] Münzenberg, G., Reisdorf, W., Hofmann. S., Agarwal Y. K., Heßberger, F. P., Poppensieker, K., Schneider, J. R. H., Schneider, W. F. W., Schmidt, K. H., Schött, H. J., Armbruster, P., Sahm, C. C., Vermeulen, D.: Z. Phys. A315 145-158 (1984)

[28] Greenwood, N. N.: Pure \& Appl. Chem. 96, 197-184 (1997)

[29] Demin, A. G., Tretyakova, S. P. Utyonkov, V. K., Shirokovsky, I. V.: Z. Phys. A315, 197-200 (1984)

[30] Randrup, J., Larsson, S. E., Möller, P., Nilsson, S. G., Pomorski, K., Sobiczewski, A.: Phys. Rev. C13, 229-239 (1976)

[31] Hofmann, S., Ninov V., Heßberger, F. P., Armbruster, P., Folger, H., Münzenberg, G., Stodel, C., Popeko, A., G., Yeremin, A., V., Saro, S., Leino, M.: to be published in Z. Phys. A 73, 624-627

[32] Münzenberg, G., Armbruster, P., Folger, H., Heßberger, F. P., Hofmann, S., Keller, J., Poppensieker, K., Reisdorf, W., Schmidt, K.- H., Schött, H. J., Leino. M. E., Hingmann, R.: Z. Phys. A317, 235-236 (1984)

[33] Münzenberg, G., Armbruster, P., Berthes, G., Folger, H. , Heßberger, F. P., Hofmann, S., Poppensieker, K., Reisdorf, W., Quint, B., Schmidt, K.- H., Schött, H. J., Sümmerer, K., Zychor, I., Leino. M. E., R., Gollerthan, U., Hanelt, E.: Z. Phys. A324, 489-490 (1987)

[34] Münzenberg, G., Armbruster, P., Berthes, G., Folger, H. , Heßberger, F. P., Hofmann, S., Keller, J., Poppensieker, K., Quint, A. B., Reisdorf, W., Schmidt, K.- H., Schött, H. J., Sümmerer, K., Zychor, I., Leino. M. E., Hingmann, R., Gollerthan, L., Hanelt, E.: Z. Phys. A328, 49-59 (1987) 
[35] Lazarev, Yu. A., Lobanov, Yu. V., Oganessian, Yu. Ts., Tsyganov, Yu. S., Utyonkov, V. K., Abdullin, F. Sh., Jliev, S., Polyakov, A. N., Rigol, J., Shirokovski, I. V., Subottin, V. G., Sukhov, A. M. Buklanov, G. V., Gikal, B. N. Kutner, V. B., Mezentzev, A. N., Sedykh, I. M., Vakatov, D. V.: Phys. Rev. Lett.

[36] Münzenberg, G., Armbruster, P., Heßberger, F. P., Hofmann S., Poppensieker, K., Reisdorf, W., Schneider J. R. H., Schneider W. F. W., Schmidt, K.-H.: Z. Phys. A309, 89-90 (1982)

[37] Münzenberg, G., Hofmann, S., Heßberger, F. P., Folger, H., Ninov, V., Poppensieker, K., Quint, A. B., Reisdorf, W., Schött, H. J., Sümmerer, K., Armbruster, P., Leino, M. E., Ackermann, D., Gollerthan, U., Hanelt, E., Morawek, W., Fujita, Y., Schwab. T., Türler. A.: Z. Phys. A330, 435-436 (1988)

[38] Lazarev, Yu. A., Lobanov, Yu. V., Oganessian, Yu. Ts., Utyonkov, V. K., Abdullin, F. Sh., Polyakov, A. N., Rigol, J., Shirokovski I. V., Tsyganov, Yu. S., Iliev, S., , Subottin, V. G., Sukhov, A. M., Buklanov, G. V., Gikal, B. N. Kutner, V. B., Mezentzev, A. N., Subotic, K.: Phys. Rev. C54 620-625 (1996) "Low Energy Scientific Publishing

[39] Ghiorso, A., Lee, D., Sommerville, L. P., Loveland, W., Nitschke, J. M., Ghiorso, W., Seaborg, G., T., Willmarth, P., Leres, R., Wydler, A., Nurmia, M., Gregorich, K., Galord, R. Hamilton, T., Hannik, N., J., Hofmann, D., C., Jarzynski, C., Kacher, D., Kadhokdayan, B., Kreek, S., Lane, M., Lyon, A., McMahan, A., Neu, M., Sikkeland, T., Swiatecki, W., Türler, A., Walton, J., T., Yashita, S.: Nucl. Phys. A583 861 (1995)

[40] Barber, R. C., Greenwood, N., N., Hryenkiewicz, A., Z., Jeannin, Y., P., Lefort, M., Sakai. M., Ulehla, I., Wapstra, A., A., Wilkinson, D. H.: Prog. Part. Nucl. Phys. 29, 453 (1992)

[41] Patyk, Z., Sobiczewski, A., Armbruster, P., Schmidt, K. H.: nucl. Phys. A491, 267 (1989)

[42] Sobiczewski, A.: Phys.-Uspekhi 39, 885-889 (1996)

[43] Cwiok, S., Pashkevich, V., V., Dudek, J., Nazarewicz, W.: Nucl. Phys. A410 254 (1983)

[44] Möller, P., Nix, J. R.: Atomic Data Nuclear Data Tables 39, 213 (1988)

[45] Möller, P., Nix, J. R.: J. Phys. G20, 1681 (1994) (1991)

[46] Smolanczuk, R.: Preprint SINS/TH/1997, Soltan Institute for Nuclear Studies, Warsaw, Poland, 1997

[47] Möller, P.: Int. Rep. GSI Darmstadt, Germany(1996) 
[48] Swiatecki, W., J.: Priv. comm (1996) Nazarewicz,

[49] Rutz, K., Bender, M., Bürvenich, T., Schilling, T., Reinhard, P.- G., Maruhn, J. A., Greiner, W.: to be published

[50] Sobiczewski, A.: Priv. comm (1996)

[51] Rigol, J.: Phys. Rev. C55, 972-973 (1997)

[52] K. Rutz, Thesis, Universität Frankfurt/Main 1997

[53] Lenske, H., Private communication 1997

[54] Heßberger, F. P., Hofmann, S., Ninov V., Armbruster, P., Folger, H., Münzenberg, Schött, H. J., Popeko, A., G., Yeremin, A., V., Andreyev, A., N., Saro, S., submitted to Z. Phys. A

[55] Swiatecki, W. J.: Nucl. Phys. A376, 275-291 (1982)

[56] Schmidt, K.-H., Morawek, W.:Rep. Prog. Phys. 54, 949-1003 (1991)

[57] Schmidt, K.-H.: Int. Worksop on Research with Fission Fragments, Benediktbeuren, Germany (1996), to be published by World Scientific Publishing Company, Singapore

[58] Bass, R. Lecture Notes in Physics 117, 420 (1980)

[59] Sandulescu, A., Gupta, R. K., Scheid, W., Greiner, W.: Phys. Lett. $60 \mathrm{~B}, 225(1976)$

[60] Singh, S., Gupta, R. K., Scheid, W., Greiner, W.: J. Phys. G18, 1243$1257(1992)$

[61] Möller, P., Nix, J. R., Armbruster. P., Hofmann, S., Münzenberg, G.: To be published

[62] Wada, T., Arimoto, Y., Tokuda, T., Ohta, M., Abe, Y.: Nucl. Phys. A616 446 (1997)

[63] Cherepanov, E. A., Adamian, G. G., Antonenko, N. V., Nasirov, A. K., Volkov, V. V.: to be published

[64] Stodel, C., Hofmann, S., Heßberger, F., P., Vinov, V., Sagaidak, R., N., Popeko, A., G., Oganessian, Yu. Ts, Lavrentjev A. Yu., Yeremin, A., V.: Ann. Rep. GSI 1996, 17 (1997)

[65] Proc. Int. conf. on Electrmagnetic Isotope Separators and Techniques Related to their Application, Bad Dürkheim Germany 1996, Eds. Münzenberg, G., Geissel, H., Scheidenberger, C.: Nucl. Instr. Meth. B126 (1997)

[66] Keller, J. G., Schmidt, K.-H., Heßberger, F. P., Münzenberg, G., Reisdorf. W., Clerc, H. G., Sahm, C. C.: Nucl. Phys. A 452 173-204 (1986) 
[67] Signorini, C.: Nucl. Phys. A616, 262 (1997)

[68] Schädel, M.: Int. Worksop on Research with Fission Fragments, Benediktbeuren, Germany (1996), to be published by World Scientific Publishing Company, Singapore, (1997)

[69] Schädel, M., Brüchle, W., Dressler, R., Eichler, B., Gäggeler, H. W., Günther, R., Gregorich, K. E., Hoffman, D. C., Hübener, S., Jost, D. T., Kratz, J. V., Paulus, W., Schumann, D., Timokhin, S., Trautmann, N., Türler, A., Wirth, G., Yakushev, A.: Nature Vol. 388, 3 July 1997

[70] Münzenberg, G. in: Experimental Techniques in Nuclear Physics, Greiner, W., Poenaru, D. N. , Eds., De Gruyter \& Co, Berlin (1997)

[71] Münzenberg, G., Faust. W., Hofmann, S., Armbruster, P., Güttner, K., Ewald. H.: Nucl. Instrum. Meth. 161, 65-82 (1979)

[72] Münzenberg, G.: Int. Journ. of Mass Spectr. Ion Phys. 14 363-378 (1974)

[73] Hofmann, S., Münzenberg, G., Heßberger, F. P., Schött, H. J.: Nucl. Instr. Meth. 223, 312-318 (1984) Energy Publishing

[74] Meier, J., Chulkov, L., Egelhof, P., Fischer, C., Henning, W., Kienlin, A., v., Kirchner, G., Kraus, G., Weinbach, A.,: Nucl. Instr. Meth. A370, 259 (1996)

[75] Fujitaka, S., Wada, M., Wang, H., Tanaka, J., Kawamaki, H., Katayama, I., Ogino, K., Katsuragawa, H., Nakamura, T., Okada, K., Ohti, S.: Nucl. Instr. Meth B126, 386 (1997)

[76] Habs, D. et al.: Int. Comm. GSI (1997) 
